$(O)$ and $(L)$ ( $\$ I$, Theorem 2$)$, the sphere $(G)$ having $G$ for center and orthogonal to $(Q)$ ( $\$ I I$, Theorem 1$)$ is the second sphere of antisimilitude of $(O)$ and $(L)$; hence $(G)$ is coaxial with these spheres.

THEOREM 3. The four spheres having for centers the vertices of a tetrahedron and orthogonal to the quasi-polar sphere cut the spheres having for diameters the respective medians of the tetrahedron along four circles belonging to the same sphere, namely, the $(G)$-sphere of the tetrahedron.

The sphere $(A)$ having $A$ for center and orthogonal to the sphere $(Q)$ is coaxial with the spheres $(G)$ and $\left(A G_{a}\right)$, for the centers of these three spheres are collinear and all three are orthogonal to $(Q)$. Similarly for the vertices $B, C, D$ of $(T)$.

UNIVERSITY OF OKLAHOMA

\title{
EUCLIDEAN CONCOMITANTS OF THE TERNARY CUBIC
}

\section{T. L. WADE}

1. Introduction; construction of concomitants. In this paper we use the results of Cramlet [1] and the writer [2] to study the euclidean concomitants of the ternary cubic curve

$$
T_{a b c} X^{a} X^{b} X^{c}=0,
$$

where $a, b, c=1,2,3$ and $T_{a b c}$ is symmetric. With tensor algebra as the medium of investigation all types of concomitants are readily constructed, and their geometric interpretations are also readily made in most cases. As is conventional in classical invariant theory, the word concomitant will be used as meaning rational integral concomitant unless stated to the contrary.

As a consequence of Theorem 3 in [2], we have the following theorem.

TheOREM I. Every euclidean concomitant of the ground form $T_{a b c} X^{a} X^{b} X^{c}(a, b, c=1,2,3)$ is expressible by composition as a tensor of order zero with the use of the coefficient tensor $T_{a b c}$, the variable coordinate tensors $X^{a}$ and $U_{a}$, and the numerical tensors $\epsilon^{a b c}, L_{a}$, and $E^{a b}$.

Presented to the Society, September 5, 1941; received by the editors of the Transsctions of this Society, September 25, 1941; accepted by them and later transferred to this Bulletin. 
The tensor $\epsilon_{a b c}$ will not appear here since we are dealing with a ground form in $X^{a}$. In detail,

$$
L_{a}=[0,0,1], \quad E^{a b}=\left[\begin{array}{lll}
1 & 0 & 0 \\
0 & 1 & 0 \\
0 & 0 & 0
\end{array}\right] .
$$

It is convenient to introduce the tensor $A^{a b}$, where

$$
A^{a b}=\epsilon^{a b c} L_{c}=\left[\begin{array}{rrr}
0 & 1 & 0 \\
-1 & 0 & 0 \\
0 & 0 & 0
\end{array}\right] \text {. }
$$

Theorem 3 of [2] is the first fundamental theorem of euclidean invariant theory in tensor form, and Theorem I above is the particular form of it which we need here; this theorem constitutes a basis for the construction of concomitants.

2. Reduction of concomitants. In [2] an algebraically complete system of euclidean invariants for the cubic $T_{a b c} X^{a} X^{b} X^{c}=0$ is given in tensor form. For the conic $C_{a b} X^{a} X^{b}=0$ the familiar algebraically complete system of three invariants is also an irreducibly complete system. But this is not true for the cubic. In this paper we shall find an irreducible system, complete through the fifth degree, of euclidean invariants for the cubic, and shall investigate geometric interpretation of these invariants in connection with other concomitants of the ground form. It is generally recognized that the problem of reduction of concomitants is more difficult than the problem of construction. See H. Weyl [3].

It is of interest to note that between the simple case of the conic and the situation for the general cubic is that of the degenerate cubic considered as the line $B_{a} X^{a}=0$ and the conic $C_{a b} X^{a} X^{b}=0$. They have the irreducibly complete system of eight invariants

$$
\begin{aligned}
& I_{1}=B_{a} B_{b} E^{a b}, \quad I_{2}=C_{a b} C_{c d} C_{e f} \epsilon^{a c e} \epsilon^{b d f}, \quad I_{3}=C_{a b} C_{c d} A^{a c} A^{b d}, \\
& I_{4}=C_{a b} E^{a b}, \quad I_{5}=C_{a b} E^{a c} E^{b d} B_{c} B_{d}, \quad I_{6}=C_{a c} C_{b d} \epsilon^{a b e} \epsilon^{c d f} B_{e} B_{f}, \\
& I_{7}=C_{a b} C_{c d} \epsilon^{a c e} A^{b d} B_{e}, \quad I_{8}=C_{a b} A^{a c} E^{b d} B_{c} B_{d} \text {. }
\end{aligned}
$$

Six of these are algebraically independent; they may be chosen as $I_{1}, \cdots, I_{6}$; then $I_{7}$ and $I_{8}$ are expressible in terms of them by means of the syzygies

$$
\begin{aligned}
3\left(I_{7}\right)^{2} & \equiv 3 I_{3} I_{6}-2 I_{1} I_{2} I_{4}-2 I_{2} I_{5} \\
\left(I_{8}\right)^{2} & \equiv I_{1} I_{4} I_{5}-\left(I_{5}\right)^{2}-\frac{1}{2}\left(I_{1}\right)^{2} I_{3} .
\end{aligned}
$$


The five invariants $I_{2}, I_{3}, I_{6}, I_{7}$, and $C_{a b} A^{a c} A^{b d} B_{c} B_{d}$ form an irreducibly complete system of affine invariants for the line and conic. They were studied recently by Weitzenböck [4], who used the symbolic notation of his text [5].

We now state the second fundamental theorem in the detailed and explicit tensor form needed to investigate the relations on the concomitants constructed in accordance with the first fundamental theorem.

THEOREM II. Every identity satisfied by the concomitants constructed by the first fundamental theorem for a set of ground forms in $X^{a}$ can be established with the basic identities:

Iden 1. $\epsilon^{a b c} E^{d e} \equiv \epsilon^{d b c} E^{a e}+\epsilon^{a d c} E^{b e}+\epsilon^{a b d} E^{c e}$,

Iden 2. $\epsilon^{a b c} X^{d} \equiv \epsilon^{d b c} X^{a}+\epsilon^{a d c} X^{b}+\epsilon^{a b d} X^{c}$,

Iden 3. $\epsilon^{a b c} \epsilon^{d e f} \equiv \epsilon^{d b c} \epsilon^{a e f}+\epsilon^{a d c} \epsilon^{b e f}+\epsilon^{a b d} \epsilon^{c e f}$,

Iden 4. $A^{a b} A^{c d} \equiv E^{a c} E^{b d}-E^{a d} E^{b c}$,

Iden $4^{\prime} . E^{a c} E^{b d} \equiv A^{a b} A^{c d}+E^{a d} E^{b c}$,

Iden 5. $A^{a b} E^{c d} \equiv A^{c b} E^{a d}+A^{a c} E^{b d}$,

Iden 6. $A^{a b} X^{c} \equiv A^{c b} X^{a}+A^{a c} X^{b}+\epsilon^{a b c} L_{d} X^{d}$,

Iden 7. $A^{a b} \epsilon^{c d e} \equiv A^{c b} \epsilon^{a d e}+A^{a c} \epsilon^{b d e}+\epsilon^{a b c} A^{d e}$,

Iden 8. $\epsilon^{a b c} A^{d e} \equiv \epsilon^{d b c} A^{a e}+\epsilon^{a d c} A^{b e}+\epsilon^{a b d} A^{c e}$,

Iden 9. $A^{a b} A^{c d} \equiv A^{c b} A^{a d}+A^{a c} A^{b d}$,

Iden 10. $E^{a b} E^{c d} E^{e f} \equiv E^{a b} E^{c_{f}} E^{e d}+E^{a d} E^{c b} E^{e f}-E^{a d} E^{c_{f}} E^{e b}-E^{a f} E^{c b} E^{e d}$ $+E^{a d} E^{c_{f}} E^{e b}$,

Iden 11. $X^{a} E^{b c} E^{d e} \equiv X^{a} E^{b e} E^{d c}+E^{a c} X^{b} E^{d e}-E^{a c} E^{b e} X^{d}-E^{a e} X^{b} E^{d c}$ $+E^{a e} E^{b c} X^{d}+\epsilon^{a b d} A^{e c} L_{f} X^{f}$.

Iden 2 may be established by expanding

and using

$$
\left|\begin{array}{llll}
\delta_{1}^{a} & \delta_{2}^{a} & \delta_{3}^{a} & X^{a} \\
\delta_{1}^{b} & \delta_{2}^{b} & \delta_{3}^{b} & X^{b} \\
\delta_{1}^{c} & \delta_{2}^{c} & \delta_{3}^{c} & X^{c} \\
\delta_{1}^{d} & \delta_{2}^{d} & \delta_{3}^{d} & X^{d}
\end{array}\right| \equiv 0
$$

$$
\epsilon^{a b c}=\left|\begin{array}{rrr}
\delta_{1}^{a} & \delta_{2}^{a} & \delta_{3}^{a} \\
\delta_{1}^{b} & \delta_{2}^{b} & \delta_{3}^{b} \\
\delta_{1}^{c} & \delta_{2}^{c} & \delta_{3}^{c}
\end{array}\right| .
$$

Most of the other identities are consequences of Iden 2 in rather obvious ways. We remark briefly about some not of this class. To obtain Iden 4 consider 


$$
A^{a b} A^{c d} \equiv \epsilon^{a b 3} \epsilon^{c d 3}=\left|\begin{array}{cc}
\delta_{1}^{a} & \delta_{2}^{a} \\
\delta_{1}^{b} & \delta_{2}^{b}
\end{array}\right| \cdot\left|\begin{array}{cc}
\delta_{1}^{c} & \delta_{1}^{d} \\
\delta_{2}^{c} & \delta_{2}^{d}
\end{array}\right| \equiv\left|\begin{array}{ll}
E^{a c} & E^{a d} \\
E^{b c} & E^{b d}
\end{array}\right|
$$

Iden 10 may be obtained from the determinantal product

$$
\left|\begin{array}{rrr}
\delta_{1}^{a} & \delta_{2}^{a} & \delta_{3}^{a} \\
\delta_{1}^{b} & \delta_{2}^{b} & \delta_{3}^{b} \\
\delta_{1}^{c} & \delta_{2}^{c} & \delta_{3}^{c}
\end{array}\right| \cdot\left|\begin{array}{ccc}
\delta_{1}^{d} & \delta_{1}^{e} & \delta_{1}^{f} \\
\delta_{2}^{d} & \delta_{2}^{e} & \delta_{2}^{f} \\
0 & 0 & 0
\end{array}\right| \equiv 0
$$

and Iden 11 from

$$
\left|\begin{array}{ccc}
\delta_{1}^{a} & \delta_{2}^{a} & \delta_{3}^{a} \\
\delta_{1}^{b} & \delta_{2}^{b} & \delta_{3}^{b} \\
\delta_{1}^{c} & \delta_{2}^{c} & \delta_{3}^{c}
\end{array}\right| \cdot\left|\begin{array}{ccc}
\delta_{1}^{d} & \delta_{1}^{e} & X^{1} \\
\delta_{2}^{d} & \delta_{2}^{e} & X^{2} \\
0 & 0 & X^{3}
\end{array}\right| \equiv \epsilon^{a b c} A^{d e} L_{f} X^{f} .
$$

That these identities constitute a reduction basis for the concomitants constructed by the first fundamental theorem may be seen by observing that they give the alternate ways of writing all types of products which arise.

3. An irreducible system, complete through the fifth degree, of euclidean invariants for the cubic $T_{a b c} X^{a} X^{b} X^{c}$. We note that the cubic has only one "formal" invariant of the first degree, $T_{a b c} \epsilon^{a b c}$, and this vanishes identically due to the skew-symmetry of $\epsilon^{a b c}$; so (i) for the cubic there is no invariant of the first degree.

We can construct three (and only three) invariants of the second degree:

$$
\begin{gathered}
A=T_{a_{1} a_{2} a_{3}} T_{b_{1} b_{2} b_{3}} E^{a_{1} a_{2}} E^{b_{1} b_{2}} E^{a_{3} b_{3}}, \quad B=T_{a_{1} a_{2} a_{3}} T_{b_{1} b_{2} b_{3}} E^{a_{1} b_{1}} E^{a_{2} b_{2}} E^{a_{3} b_{3}}, \\
C=T_{a_{1} a_{2} a_{3}} T_{b_{1} b_{2} b_{3}} A^{a_{1} b_{1}} A^{a_{2} b_{2}} E^{a_{3} b_{3}}
\end{gathered}
$$

Invariants as $T_{a_{1} a_{2} a_{3}} T_{b_{1} b_{2} b_{3}} A^{a_{1} b_{1}} A^{a_{2} b_{2}} A^{a_{3} b_{3}}$ and $T_{a_{1} a_{2} a_{3}} T_{b_{1} b_{2} b_{3}} A^{a_{1} a_{2}} E^{b_{1} b_{2}} E^{a_{3} b_{3}}$ which vanish identically on the interchange of equivalent indices are not listed here, nor in similar circumstances in the future. In this connection one should keep in mind the skew-symmetry of $\epsilon^{a b c}$ and of $A^{a b}$. The only identities applicable to $A$ and $B$ are Idens $4^{\prime}$ and 10 . The application of the latter to each of these merely gives $A \equiv A$ and $B \equiv B$. The application of Iden $4^{\prime}$ to either of them results in $C \equiv A-B$. The only identities applicable to $C$ are Idens 4 and 5 . The first results in the relation just given, and the latter in $C \equiv C$. Thus $C \equiv A-B$ is the only relation on the invariants $A, B, C$. Therefore, (ii) for the cubic there are two irreducible invariants of the second degree, and these may be chosen as 
$I I_{1}=T_{a_{1} a_{2} a_{3}} T_{b_{1} b_{2} b_{3}} E^{a_{1} a_{2}} E^{b_{1} b_{2}} E^{a_{3} b_{3}}, \quad I I_{2}=T_{a_{1} a_{2} a_{3}} T_{b_{1} b_{2} b_{3}} E^{a_{1} b_{1}} E^{a_{2} b_{2}} E^{a_{3} b_{3}}$.

Often we shall use the contracted notation

$$
{ }_{(k)} T=T_{a_{11} a_{12} a_{13}} T_{a_{21} a_{22} a_{23}} \cdots T_{a_{k 1} a_{k 2} a_{k 3}} .
$$

To illustrate, $I I_{1}={ }_{(2)} T \cdot E^{a_{1} a_{2}} E^{b_{1} b_{2}} E^{a_{3} b_{3}}$.

There are three invariants of the third degree to consider:

$$
\begin{gathered}
A={ }_{(3)} T \cdot A^{a_{1} b_{1}} E^{b_{2} c_{1}} A^{c_{2} a_{2}} \epsilon^{a_{3} b_{3} c_{3}}, \quad B={ }_{(3)} T \cdot E^{a_{1} c_{1}} E^{b_{1} b_{2}} A^{c_{2} a_{2}} \epsilon^{a_{3} b_{3} c_{3}}, \\
C={ }_{(3)} T \cdot A^{a_{1} b_{1}} A^{b_{2} c_{1}} A^{c_{2} a_{2}} \epsilon^{a_{3} b_{3} c_{3}} .
\end{gathered}
$$

Applying Iden 5 to $A^{c_{2} a_{2}} E^{b_{1} b_{2}}$ of $B$, we get $B=-A-A$, or $B=-2 A$. Applying Iden 4 to $A^{a_{1} b_{1}} A^{b_{2} c_{1}}$ of $C$, we obtain $C=A-B$. On application of the appropriate reduction formulas in all possible ways to $A, B, C$, it is found that there is no relation on these expressions other than the two given. Hence: (iii) For the cubic there is one irreducible invariant of the third degree, and this may be chosen as

$$
I I I=(1 / 6) T_{a_{1} a_{2} a_{3}} T_{b_{1} b_{2} b_{3}} T_{c_{1} c_{2} c_{3}} A^{a_{1} b_{1}} A^{b_{2} c_{1}} A^{a_{2} c_{2}} \epsilon^{a_{3} b_{3} c_{3}} .
$$

The number of formal invariants which one can construct increases rapidly as the degree goes beyond three. Their consideration is facilitated by considering all invariants of a certain type together. (iv-1) For the cubic there is only one invariant of the fourth degree which contains the numerical tensor $\epsilon^{a b c}$ four times, and this is irreducible, it being the well known projective invariant of the fourth degree

$$
I V_{1}={ }_{(4)} T \cdot \epsilon^{a_{1} b_{1} c_{1}} \epsilon^{a_{2} b_{2} d_{1}} \epsilon^{a_{3} c_{2} d_{2}} \epsilon^{b_{3} c_{3} d_{3}} .
$$

There are six invariants of the fourth degree which contain factors like $\epsilon^{a b c}$ twice, the remaining factors being like $E^{a b}$ :

$$
\begin{aligned}
& A={ }_{(4)} T \cdot \epsilon^{a_{1} b_{1} c_{1}} \epsilon^{a_{2} b_{2} c_{2}} E^{a_{3} d_{1}} E^{b_{3} d_{2}} E^{c_{3} d_{3}}, \quad B={ }_{(4)} T \cdot \epsilon^{a_{1} b_{1} c_{1}} \epsilon^{a_{2} b_{2} c_{2}} E^{a_{3} b_{3}} E^{c_{3} d_{1}} E^{d_{2} d_{3}}, \\
& C={ }_{(4)} T \cdot \epsilon^{a_{1} b_{1} c_{1}} \epsilon^{b_{2} c_{2} d_{1}} E^{a_{2} a_{3}} E^{b_{3} c_{3}} E^{d_{2} d_{3}}, \quad D={ }_{(4)} T \cdot \epsilon^{a_{1} b_{1} c_{1}} \epsilon^{b_{2} c_{2} d_{1}} E^{a_{2} a_{3}} E^{b_{3} d_{2}} E^{c_{3} d_{3}},
\end{aligned}
$$

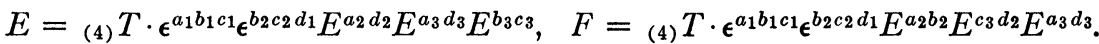

Applying the basic identities in all possible ways, we find that these invariants are connected by the relations

$A \equiv D+2 F ; \quad B \equiv E+2 F ; \quad B \equiv C+2 D ; \quad C \equiv 2 D+E-2 F ;$

and only these. Therefore: (iv-2) For the cubic there are two irreducible invariants of the fourth degree which contain factors like $\epsilon^{a b c}$ twice, the remaining factors being like $E^{a b}$, and these may be chosen as 


$$
\begin{aligned}
& I V_{2}={ }_{(4)} T \cdot \epsilon^{a_{1} b_{1} c_{1}} \epsilon^{b_{2} c_{2} d_{1}} E^{a_{2} a_{3}} E^{b_{3} c_{3}} E^{d_{2} d_{3}}, \\
& I V_{3}={ }_{(4)} T \cdot \epsilon^{a_{1} b_{1} c_{1}} \epsilon^{b_{2} c_{2} d_{1}} E^{a_{2} b_{2}} E^{c_{3} d_{2}} E^{a_{3} d_{3}} .
\end{aligned}
$$

Like considerations lead us to the conclusions: (iv-3) For the cubic there is one irreducible invariant of the fourth degree which contains factors like $\epsilon^{a b c}$ twice, the other factors being like $E^{a b}$, and this may be chosen as

$$
I V_{4}={ }_{(4)} T \cdot \epsilon^{a_{1} b_{1} c_{1}} \epsilon^{a_{2} b_{2} c_{2}} E^{b_{3} c_{3}} E^{d_{1} d_{2}} A^{a_{3} d_{3}} .
$$

(iv-4) For the cubic there is one irreducible invariant of the fourth degree whose numerical tensor product is composed entirely of factors like $E^{a b}$, except for one factor like $A^{a b}$, and this may be chosen as

$$
I V_{5}={ }_{(4)} T \cdot E^{a_{1} a_{2}} A^{a_{3} b_{1}} E^{b_{2} c_{2}} E^{b_{3} c_{1}} E^{c_{3} d_{1}} E^{d_{2} d_{3}} .
$$

For invariants of the fourth degree constructed wholly with the aid of $E^{a b}$, there are five possibilities:

$$
\begin{aligned}
& A={ }_{(4)} T \cdot E^{a_{1} a_{2}} E^{a_{3} b_{1}} E^{b_{2} c_{1}} E^{b_{3} c_{2}} E^{c_{3} d_{1}} E^{d_{2} d_{3}}, \\
& B={ }_{(4)} T \cdot E^{a_{1} b_{1}} E^{a_{2} b_{2}} E^{a_{3} c_{1}} E^{b_{3} d_{1}} E^{c_{2} d_{2}} E^{c_{3} d_{3}}, \\
& C={ }_{(4)} T \cdot E^{a_{1} b_{1}} E^{a_{2} c_{1}} E^{a_{3} d_{1}} E^{b_{2} c_{2}} E^{b_{3} d_{2}} E^{c_{3} d_{3}}, \\
& D={ }_{(4)} T \cdot E^{a_{1} a_{2}} E^{a_{3} b_{1}} E^{b_{2} c_{1}} E^{b_{3} d_{1}} E^{c_{2} d_{2}} E^{c_{3} d_{3}}, \\
& E={ }_{(4)} T \cdot E^{a_{1} a_{2}} E^{a_{3} b_{1}} E^{b_{2} c_{1}} E^{b_{3} d_{1}} E^{c_{2} c_{3}} E^{d_{2} d_{3}}
\end{aligned}
$$

Applying the identities 1-11 in all possible ways it is found that there are four and only four relations on these expressions; so: (iv-5) For the cubic there is only one irreducible invariant constructed wholly with the aid of $E^{a b}$, of the fourth degree, and this may be chosen as

$$
I V_{6}={ }_{(4)} T \cdot E^{a_{1} a_{2}} E^{a_{2} b_{1}} E^{b_{2} c_{1}} E^{b_{3} c_{2}} E^{c_{3} d_{1}} E^{d_{2} d_{3}} .
$$

Then

$$
\begin{aligned}
& B \equiv I V_{6}+(1 / 2)\left(I I_{2}\right)^{2}-(1 / 2)\left(I I_{1}\right)^{2}, \\
& C \equiv I V_{6}+I I_{1} \cdot I I_{2}-\left(I I_{1}\right)^{2}, \\
& D \equiv I V_{6}+(1 / 2) I I_{1} \cdot I I_{2}-(1 / 2)\left(I I_{1}\right)^{2}, \\
& E \equiv I V_{6}+(1 / 2)\left(I I_{1}\right)^{2}-(1 / 2) I I_{1} \cdot I I_{2} .
\end{aligned}
$$

All other invariants of the fourth degree are found to be reducible; so we may summarize as follows: (iv) For the cubic there are six irreducible invariants of the fourth degree, and these may be chosen as $I V_{1}, I V_{2}, I V_{3}, I V_{4}, I V_{5}, I V_{6}$. 
In a similar manner it is found that: (v) For the cubic there are two irreducible invariants of the fifth degree, and these may be chosen as

$$
\begin{aligned}
& V_{1}={ }_{(5)} T \cdot \epsilon^{a_{1} b_{1} c_{1}} \epsilon^{a_{2} b_{2} c_{2}} \epsilon^{a_{3} d_{1} e_{1}} A^{d_{2} e_{2}} E^{b_{3} c_{3}} E^{d_{3} e_{3}}, \\
& V_{2}={ }_{(5)} T \cdot \epsilon^{a_{1} b_{1} c_{1}} \epsilon^{a_{2} b_{2} c_{2} \epsilon^{a_{3}} \epsilon_{1} e_{1}} A^{b_{3} e_{2}} E^{d_{2} d_{3}} A^{c_{3} e_{3}} .
\end{aligned}
$$

A typical reduction is that of $A={ }_{(5)} T \cdot \epsilon^{d_{1} b_{1 c} c_{1}} A^{a_{1} b_{2}} E^{a_{2} d_{2}} E^{c_{22} c_{3}} E^{b_{3} d_{3}} E^{a_{3 e} e_{1}} E^{e_{22} e_{3}}$. Iden 5 applied to $A^{a_{1} b_{2}} E^{d_{2} a_{2}}$ of $A$ gives $A \equiv 4 I I_{1} \cdot I I I-A$, or $A \equiv 2 I I_{1} \cdot I I I$.

We may combine (i), (ii), (iii), (iv), (v) in the following theorem.

Theorem III. For the cubic $T_{a b c} X^{a} X^{b} X^{c}(a, b, c=1,2,3)$ there are eleven irreducible euclidean invariants of degree less than six, and these may be chosen as $I I_{1}, I I_{2}, I I I, I V_{1}, I V_{2}, I V_{3}, I V_{4}, I V_{5}, I V_{6}, V_{1}, V_{2}$.

4. An irreducible system, complete through the fourth degree, of euclidean covariants for the cubic. There seems to have been no systematic study of euclidean covariants for the cubic curve of the third order. The best known are the Hessian, the polar conic of the line at infinity, and the Laplacian. We shall of ten speak of a covariant of degree $i$ in $T_{a b c}$ and order $j$ in $X^{a}$ as a $(i, j)$ covariant. The line at infinity is a $(0,1)$ covariant, having for its equation $\&=L_{a} X^{a}=X^{3}=0$.

4.1. $(i, 1)$ covariants. For the cubic $T_{a b c} X^{a} X^{b} X^{c}$ there is only one $(1,1)$ covariant, and this is $L_{1}=T_{a b c} E^{a b} X^{c}$. There is no $(2,1)$ covariant. There are four irreducible $(3,1)$ covariants, and these may be chosen as

$$
\begin{array}{ll}
L_{31}={ }_{(3)} T \cdot \epsilon^{a_{1} b_{1} c_{1}} \epsilon^{a_{2} b_{2} c_{2}} E^{b_{3} c_{3}} X^{a_{3}}, & L_{32}={ }_{(3)} T \cdot E^{a_{1} b_{1}} E^{a_{2} c_{1}} E^{c_{2} c_{3}} E^{b_{2} b_{3}} X^{a_{3}}, \\
L_{33}={ }_{(3)} T \cdot A^{a_{1} b_{1}} E^{a_{2} c_{1}} E^{b_{2} c_{2}} E^{b_{3} c_{3}} X^{a_{3}}, & L_{34}={ }_{(3)} T \cdot A^{a_{1} b_{1}} E^{a_{2} c_{1}} E^{b_{2} b_{3}} E^{c_{1} c_{2}} X^{a_{3}} .
\end{array}
$$

The other $(3,1)$ covariants

$$
\begin{array}{ll}
A={ }_{(3)} T \cdot E^{a_{1} b_{1}} E^{a_{2} c_{1}} E^{b_{2} c_{2}} E^{b_{3} c_{3}} X^{a_{3}}, & B={ }_{(3)} T \cdot E^{a_{1} b_{1}} E^{a_{2} b_{2}} E^{b_{3} c_{1}} E^{c_{2} c_{3}} X^{a_{3}}, \\
C={ }_{(3)} T \cdot A^{a_{1} b_{1}} E^{a_{2} b_{2}} E^{b_{3} c_{1}} E^{c_{2} c_{3}} X^{a_{3}}, & D={ }_{(3)} T \cdot E^{a_{1} b_{1}} E^{a_{2} b_{2}} A^{b_{3} c_{1}} E^{c_{2} c_{3}} X^{a_{3}},
\end{array}
$$

are expressible in terms of the irreducible ones by the relations:

$$
\begin{aligned}
& A \equiv L_{32}+\left(I I_{2}-I I_{1}\right)+2 I I I \cdot R, \\
& B \equiv L_{32}+(1 / 2)\left(I I_{2}-I I_{1}\right) L_{1}+2 I I I \cdot R, \\
& C \equiv L_{33}, \\
& D \equiv L_{34}-L_{33} .
\end{aligned}
$$

In like manner it is found that there is only one irreducible $(4,1)$ covariant, and this may be chosen as

$$
L_{4} \equiv{ }_{(4)} T \cdot \epsilon^{a_{1} b_{1} c_{1}} E^{a_{2} b_{2}} E^{d_{1} d_{2}} E^{b_{3} c_{2}} E^{c_{3} d_{3}} X^{a_{3}} .
$$


Therefore we have this theorem.

THEOREM IV. For the cubic there are seven irreducible $(i, 1)$ euclidean covariants $(i=0,1,2,3,4)$, and these may be chosen as $\&, L_{1}, L_{31}, L_{32}$, $L_{33}, L_{34}$, and $L_{4}$.

4.2. $(i, 2)$ covariants. Obviously there is no $(1,2)$ covariant. There are four $(2,2)$ covariants :

$$
\begin{array}{ll}
A={ }_{(2)} T \cdot E^{a_{1} b_{1}} E^{a_{2} b_{2}} X^{a_{3}} X^{b_{3}}, & B={ }_{(2)} T \cdot A^{a_{1} b_{1}} A^{a_{2} b_{2}} X^{a_{3}} X^{b_{3}}, \\
C={ }_{(2)} T \cdot A^{a_{1} b_{1}} E^{b_{2} b_{3}} X^{a_{2}} X^{a_{3}}, & D={ }_{(2)} T \cdot E^{a_{1} b_{1}} E^{b_{2} b_{3}} X^{a_{2}} X^{a_{3}} .
\end{array}
$$

The expressions $C$ and $D$ contain no significant reducible factor, and consequently are irreducible. Iden 4 applied to $A^{a_{1} b_{1}} A^{a_{2} b_{2}}$ gives $B \equiv\left(L_{1}\right)^{2}-A$. This is the only relation that exists on $A$ and $B$; so (i) there are three irreducible $(2,2)$ covariants, and these may be chosen as

$$
\begin{gathered}
C_{21}={ }_{(2)} T \cdot A^{a_{1} b_{1}} A^{a_{2} b_{2}} X^{a_{3}} X^{b_{3}}, \quad C_{22}={ }_{(2)} T \cdot A^{a_{1} b_{1}} E^{b_{2} b_{3}} X^{a_{2}} X^{a_{3}}, \\
C_{23}={ }_{(2)} T \cdot E^{a_{1} b_{1}} E^{b_{2} b_{3}} X^{a_{2}} X^{a_{3}} .
\end{gathered}
$$

One may construct three $(3,2)$ covariants

$$
\begin{gathered}
A={ }_{(3)} T \cdot \epsilon^{a_{1} b_{1} c_{1}} A^{a_{2} b_{2}} E^{c_{2} c_{3}} X^{a_{3}} X^{b_{3}}, \quad B={ }_{(3)} T \cdot \epsilon^{a_{1} b_{1} c_{1}} A^{a_{2} c_{2}} E^{b_{2} c_{3}} X^{a_{3}} X^{b_{3}}, \\
C={ }_{(3)} T \cdot \epsilon^{a_{1} b_{1} c_{1}} A^{b_{2} c_{2}} E^{b_{3} c_{3}} X^{a_{2}} X^{a_{3}} .
\end{gathered}
$$

Applying Iden 5 to $A^{a_{2} b_{2}} C^{c_{2} c_{3}}$ of $A$ we obtain $A \equiv 2 B$. Iden 6 applied to $A^{a_{2 c 2}} X^{b_{3}}$ of $B$ gives $B \equiv C-B-L_{31} \cdot \Omega$, or $2 B \equiv C-L_{31} \cdot \Omega$. There is no other relation on these expressions. Therefore (ii) there is one irreducible $(3,2)$ covariant, and this may be chosen as

$$
C_{3}={ }_{(3)} T \cdot \epsilon^{a_{1} b_{1} c_{1}} A^{b_{2} c_{2}} E^{b_{3} c_{3}} X^{a_{2}} X^{a_{3}} .
$$

(iii) By a similar procedure it is found that there are five irreducible $(4,2)$ covariants, and these may be chosen as

$$
\begin{aligned}
& C_{41}={ }_{(4)} T \cdot \epsilon^{a_{1} b_{1} c_{1}} \epsilon^{a_{2} b_{2} c_{2}} E^{c_{3} d_{1}} E^{d_{2} d_{3}} X^{a_{3}} X^{b_{3}}, \\
& C_{42}={ }_{(4)} T \cdot \epsilon^{a_{1} b_{1} c_{1}} \epsilon^{a_{2} b_{2} d_{1}} E^{c_{2} c_{3}} E^{d_{2} d_{3}} X^{a_{3}} X^{b_{3}}, \\
& C_{43}={ }_{(4)} T \cdot \epsilon^{b_{1} c_{1} d_{1}} \epsilon^{b_{2} c_{2} d_{2}} E^{a_{1} b_{3}} E^{c_{3} d_{3}} X^{a_{2}} X^{a_{3}}, \\
& C_{44}={ }_{(4)} T \cdot \epsilon^{a_{1} b_{1} c_{1}} \epsilon^{a_{2} b_{2} c_{2}} E^{d_{1} d_{2}} A^{c_{3} d_{3}} X^{a_{3}} X^{b_{3}}, \\
& C_{45}={ }_{(4)} T \cdot \epsilon^{b_{1} c_{1} d_{1}} \epsilon^{b_{2} c_{2} d_{2}} E^{c_{3} d_{3}} A^{b_{3} a_{1}} X^{a_{2}} X^{a_{3}} .
\end{aligned}
$$

Combining (i), (ii), and (iii) we have this theorem

Theorem V. For the cubic there are nine irreducible $(i, 2)$ euclidean covariants $(i=1,2,3,4)$, and these may be chosen as $C_{21}, C_{22}, C_{23}, C_{3}$, $C_{41}, C_{42}, C_{43}, C_{44}, C_{45}$. 
Using the general methods of construction and reduction as we have above, we find that this theorem follows.

Theorem VI. For the cubic $(i=1,2,3,4)$ : (i) there are six euclidean irreducible $(i, 3)$ covariants, and these may be chosen as

$$
\begin{aligned}
T_{31} & ={ }_{(3)} T \cdot \epsilon^{a_{1} b_{1} c_{1}} \epsilon^{a_{2} b_{2} c_{2}} X^{a_{3}} X^{b_{3}} X^{c_{3}}, \\
T_{32} & ={ }_{(3)} T \cdot A^{a_{1} b_{1}} E^{b_{2} c_{1}} E^{c_{2} c_{3}} X^{b_{3}} X^{a_{2}} X^{a_{3}}, \\
T_{33} & ={ }_{(3)} T \cdot A^{b_{1} c_{1}} E^{b_{2} c_{2}} E^{a_{1} c_{3}} X^{b_{3}} X^{a_{2}} X^{a_{3}}, \\
T_{41} & ={ }_{(4)} T \cdot \epsilon^{a_{1} b_{1} c_{1}} E^{b_{2} d_{1}} E^{c_{2} d_{2}} E^{c_{3} d_{3}} X^{b_{3}} X^{a_{2}} X^{a_{3}}, \\
T_{42} & ={ }_{(4)} T \cdot \epsilon^{d_{1} b_{1} c_{1}} E^{b_{2} c_{2}} E^{a_{1} d_{2}} E^{c_{3} d_{3}} X^{b_{3}} X^{a_{2}} X^{a_{3}}, \\
T & =T_{a b c} X^{a} X^{b} X^{c} .
\end{aligned}
$$

(ii) There are three irreducible $(i, 4)$ covariants, and these may be chosen as

$$
\begin{gathered}
Q_{2}={ }_{(2)} T \cdot E^{a_{1} b_{1}} X^{a_{2}} X^{a_{3}} X^{b_{2}} X^{b_{3}}, Q_{41}={ }_{(4)} T \cdot \epsilon^{b_{1} c_{1} d_{1}} \epsilon^{b_{2} c_{2} d_{2}} E^{a_{1} d_{3}} X^{a_{2}} X^{b_{2}} X^{c_{3}}, \\
Q_{42}={ }_{(4)} T \cdot \epsilon^{b_{1} c_{1} d_{1}} \epsilon^{b_{2} c_{2} d_{2}} A^{a_{1} d_{3}} X^{a_{2}} X^{a_{3}} X^{b_{3}} X^{c_{3}} .
\end{gathered}
$$

(iii) There are two irreducible quintics, and these may be chosen as

$$
\begin{aligned}
& R_{3}={ }_{\left.{ }_{3}\right)} T \cdot E^{a_{1} c_{1}} A^{b_{1} c_{2}} X^{a_{2}} X^{a_{3}} X^{b_{2}} X^{b_{3}} X^{c_{3}}, \\
& R_{4}={ }_{(4)} T \cdot \epsilon^{a_{1} c_{1} d_{1}} E^{b_{1} c_{2}} E^{d_{2} d_{3}} X^{a_{2}} X^{a_{3}} X^{b_{2}} X^{b_{3}} X^{c_{3}} .
\end{aligned}
$$

(iv) There is no irreducible covariant for $i<5, j>6$.

5. Geometric interpretations. It should be understood that the general cubic curve given by the general equation of the third degree in $X^{a}$,

$$
T=T_{a b c} X^{a} X^{b} X^{c}=0,
$$

( $a, b, c=1,2,3$ and $T_{a b c}$ symmetric) is under consideration here. For such cubic no invariant is zero, and no covariant vanishing identically, unless specifically stated so. The point $P_{2}=T_{a_{1} a_{2} a_{3}} T_{b_{1} b_{2} b_{3}}$ $\cdot \epsilon^{a_{1} b_{1} r} A^{a_{2} b_{2}} E^{a_{3} b_{3}} U_{r}=0$ in expanded form is

$$
\begin{aligned}
& \left(T_{112} T_{123}+T_{122} T_{223}-T_{122} T_{113}-T_{123} T_{222}\right) U_{1} \\
& \left(T_{122} T_{123}+T_{112} T_{113}-T_{112} T_{223}-T_{111} T_{123}\right) U_{2} \\
& \quad\left(T_{111} T_{112}-\left(T_{112}\right)^{2}-\left(T_{122}\right)^{2}+T_{112} T_{222}\right) U_{3}=0 .
\end{aligned}
$$

Keeping this expanded form in mind, and examining the contents of the paper by Thomae [6], or that by Stuyvaert [7], we observe the truth of this theorem.

Theorem VII. $P_{2}$ is the unique point whose polar conic is a circle. 
When we speak of the polar of a point (or of the pole of a line), unless specified to the contrary, we mean with respect to the fundamental cubic $T$.

TheOREM VIII. (a) The polar conic of $P_{2}$ is $C_{3}$, and (b) $C_{3}$ is a circle.

Obviously the polar conic of $Y^{a}$ with respect to $T$ has the equation $T_{a b c} Y^{a} X^{b} X^{c}=0$. Let $Y^{r} U_{r}=T_{a_{1} a_{2} a_{3}} T_{b_{1} b_{2} b_{3}} \epsilon^{a_{1} b_{1} r} A^{a_{2} b_{2}} E^{a_{3} b_{3}} U_{r}$. Then (a) is evident, and (b) follows.

THEOREM IX. Every covariant conic of the cubic $T$ whose coefficients are of the third degree in $T_{a b c}$ is a circle.

In $\$ 4.2$ it was shown that one can construct three $(3,2)$ covariants, there designated by $A, B, C$, and that these are connected by the relations $A \equiv 2 B$, and $2 B \equiv C-L_{31} \cdot \Omega$. By Theorem VIII, $C_{3}$ is a circle, and as a consequence of the relations just given $A$ and $B$ are also circles. Recall from $\$ 4$ that there are no covariant conics of degree less than two, that there are covariant lines of the first and third degree, but none of the second, and further from $\$ 3$ there is no invariant of degree less than two. As a consequence of these facts we conclude that every covariant conic $Q$ of the third degree is of the form $Q \equiv k_{1} C_{3}+k_{2} R$, where $k_{1}$ and $k_{2}$ are constants, and $R$ is at most linear in $X^{a}$. But since $C_{3}$ is a circle, every such conic $Q$ is a circle.

The condition that the polar conic of the point $Y^{a}, T_{a b c} X^{a} X^{b} Y^{c}$, be a rectangular hyperbola is that $\left(T_{11 c}+T_{22 c}\right) Y^{c}=T_{a b c} E^{a b} Y^{c}=0$.

TheOREM X. The line $L_{1}=0$ is the locus of points whose polar conics are rectangular hyperbolas.

This line is called the Laplacian of the cubic. The cubic for which $L_{1} \equiv 0$ has been studied by Brooks [8].

Theorem $\mathrm{X}^{\prime}$. The Laplacian of the Hessian $H={ }_{(3)} T \cdot \epsilon^{a_{1} b_{1} c_{1}} \epsilon^{a_{2} b_{2} c_{2}}$ - $X^{a_{3}} X^{b_{3}} X^{c_{3}}=0$ is $L_{31}=0$.

Adapting the argument of White [9] to tensor notation, we find that the equation of the polar conic of the line $V_{a} X^{a}=0$ with respect to $T$ is

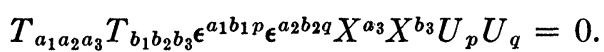

If in this we replace $U_{p}$ by $L_{p}=[0,0,1]$ and use $A^{a b}=\epsilon^{a b c} L_{c}$, we have this next theorem.

THEOREM XI. The polar conic of the line at infinity is $C_{21}=0$.

In like manner, we have this theorem. 
TheOREM XI'. The polar conic of the Laplacian is $C_{42}=0$.

The polar line of the point $Y^{r} U_{r}=0$ with respect to $C_{21}=0$ is

$$
T_{a_{1} a_{2} a_{3}} T_{b_{1} b_{2} b_{3}} A^{a_{1} b_{1}} A^{a_{2} b_{2}} X^{a_{3}} X^{a_{3}} Y^{b_{3}}=0 .
$$

In particular let $Y^{r} U_{r}=0$ be $P_{2}$. We have for the polar of $P_{2}$ with respect to $C_{21}$

$$
\begin{aligned}
K & =T_{a_{1} a_{2} a_{3}} T_{b_{1} b_{2} b_{3}} T_{c_{1} c_{2} c_{3}} T_{d_{1} d_{2} d_{3}} A^{a_{1} b_{1}} A^{a_{2} b_{2}} \epsilon^{c_{1} d_{1} b_{1}} A^{c_{2} d_{2}} E^{c_{3} d_{3}} X^{a_{3}} \\
& =4 I I I \cdot L_{1}-{ }_{(4)} T \cdot \epsilon^{d_{1} b_{1} c_{1}} A^{d_{2} b_{2}} E^{d_{3} b_{3}} E^{a_{2} c_{2}} E^{a_{3} c_{3}} X^{a_{1}} \text { (by Iden 4). }
\end{aligned}
$$

Using other identities we find that the last term is $2 I I I \cdot L_{1}$; so $K \equiv 2 I I I \cdot L_{1}$.

Theorem XII. The Laplacian of a cubic is the polar with respect to the polar conic of the line at infinity of the unique point whose polar conic is a circle.

Rather evident are these theorems and corollary.

TheOREM XIII. The polar conic of $T_{a b c} E^{a b} A^{c r} U_{r}=0$, the point at infinity on the Laplacian, is $C_{22}=0$.

Corollary. $C_{22}=0$ is a rectangular hyperbola.

THEOREM XIV. The polar conic of $T_{a b c} E^{a b} E^{c r} U_{r}=0$, the point at infinity in the direction perpendicular to the Laplacian, is $C_{23}=0$.

Taking the polar of $T_{a b c} E^{a b} E^{c r} U_{r}=0$ and $T_{a b c} E^{a b} A^{\text {cr }} U_{r}=0$, respectively, with respect to $C_{23}=0$, we get these theorems.

THEOREM XV. The diameter of the conic $C_{23}$ conjugate to the direction perpendicular to the Laplacian is $L_{32}=0$.

Theorem XVI. The diameter of the conic $C_{23}$ conjugate to the direction of the Laplacian is $L_{34}=0$.

The condition for two lines $V_{a}$ and $W_{a}$ to be perpendicular is that $V_{a} W_{b} E^{a b}=0$, and the condition for them to be parallel is that $V_{a} W_{b} A^{a b}=0$. From these a number of geometric facts follow quite directly:

A.1. The locus of points whose linear polars are parallel to the Laplacian is the conic $C_{22}=0$.

2. The locus of points whose linear polars are perpendicular to the Laplacian is the conic $C_{23}=0$.

3. The locus of points whose linear polars with respect to the Hessian are perpendicular to the Laplacian is the conic $C_{41}=0$.

4. The locus of points whose linear polars with respect to the Hessian are parallel to the Laplacian is the conic $C_{44}=0$. 
5. The locus of points whose linear polars are perpendicular to the Laplacian of the Hessian is the conic $C_{43}=0$.

6. The locus of points whose linear polars are parallel to the Laplacian of the Hessian is the conic $C_{45}=0$.

7. The locus of points whose linear polars with respect to the fundamental cubic and the Hessian are perpendicular is the quartic $Q_{41}=0$.

8. The locus of points whose linear polars with respect to the fundamental cubic and the Hessian are parallel is the quartic $Q_{42}=0$.

9. The locus of points whose linear polars with respect to fundamental cubic and the quartic $Q_{2}$ are parallel is the quintic $R_{3}=0$.

10. The Laplacian of the fundamental cubic and the Laplacian of the Hessian are parallel if (and only if) $I V_{4}=0$, and they are perpendicular if

(4) $T \cdot \epsilon^{a_{1} b_{1} c_{1}} \epsilon^{a_{2} b_{2} c_{2}} E^{b_{3} c_{3}} E^{a_{3} d_{1}} E^{d_{2} d_{3}} \equiv I V_{2}+2 I V_{3}=0$.

11. The Laplacian and $L_{32}=0$ are parallel if $I V_{5}=0$, and they are perpendicular if

(4) $T \cdot E^{a_{1} a_{2}} E^{a_{3} b_{1}} E^{b_{2} c_{2}} E^{b_{3} c_{3}} E^{c_{1} d_{1}} E^{d_{2} d_{3}} \equiv I V_{6}+(1 / 2)\left(I I_{1}\right)^{2}-(1 / 2) I I_{1} \cdot I I_{2}=0$.

The line $V_{a} X^{a}=0$ is said to be minimal if $V_{a} V_{b} E^{a b}=0$. Hence we have the following facts.

B.1. The Laplacian is a minimal line if (and only if) $I I_{1}=0$.

2. The locus of points whose linear polars are minimal lines is the quartic $Q_{2}=0$.

Using the condition for incidence of the point $V_{a}$ and the line $Y^{a}$, namely that $V_{a} Y^{a}=0$, we get these statements.

C.1. $P_{2}$, the unique point whose polar conic is a circle, lies on the Laplacian if $I I I=0$.

2. $P_{2}$ lies on the Laplacian of the Hessian if $V_{1}=0$.

Not so direct is the next fact.

3. The line through $P_{2}$ and perpendicular to the Laplacian is $L_{33}=0$.

6. Some concomitants in expanded form. We list a few typical concomitants in expanded form. These are obtained by carrying out the indicated summations in the tensor-invariant forms of the concomitants, using the defined values of the components of $\epsilon^{a b c}, A^{a b}$, and $E^{a b}$.

$$
\begin{aligned}
I I_{1} & =T_{a_{1} a_{2} a_{3}} T_{b_{1} b_{2} b_{3}} E^{a_{1} a_{2}} E^{b_{1} b_{2}} E^{a_{3} b_{3}}=\left(T_{111}+T_{122}\right)^{2}+\left(T_{112}+T_{222}\right)^{2}, \\
I I_{2} & =T_{a_{1} a_{2} a_{3}} T_{b_{1} b_{2} b_{3}} E^{a_{1} b_{1}} E^{a_{2} b_{2}} E^{a_{3} b_{3}} \\
& =\left(T_{111}\right)^{2}+3\left(T_{112}\right)^{2}+3\left(T_{122}\right)^{2}+\left(T_{222}\right)^{2},
\end{aligned}
$$




$$
\begin{aligned}
& I I I=(1 / 6) T_{a_{1} a_{2} a_{3}} T_{b_{1} b_{2} b_{3}} T_{c_{1} c_{2} c_{3}} A^{a_{1} b_{1}} A^{b_{2} c_{1}} A^{a_{2} c_{2}} \epsilon^{a_{3} b_{3} c_{3}} \\
& =\left|\begin{array}{lll}
T_{111} & T_{112} & T_{113} \\
T_{211} & T_{212} & T_{213} \\
T_{221} & T_{222} & T_{223}
\end{array}\right|, \\
& I V_{5}={ }_{(4)} T \cdot E^{a_{1} a_{2}} A^{a_{3} b_{1}} E^{b_{2} c_{1}} E^{b_{3} c_{2}} E^{c_{3} d_{1}} E^{d_{2} d_{3}} \\
& =3\left(T_{111}\right)^{2} T_{112} T_{122}+2\left(T_{122}\right)^{3} T_{222}+3 T_{111}\left(T_{122}\right)^{2} T_{222} \\
& +6 T_{111} T_{112}\left(T_{122}\right)^{2}+T_{111}\left(T_{222}\right)^{3}+3 T_{112}\left(T_{122}\right)^{3} \\
& -3 T_{112} T_{122}\left(T_{222}\right)^{2}-2 T_{111}\left(T_{112}\right)^{3}-3 T_{111}\left(T_{112}\right)^{2} T_{222} \\
& -6\left(T_{112}\right)^{2} T_{122} T_{222}-\left(T_{111}\right)^{3} T_{222}-3\left(T_{112}\right)^{3} T_{122} \text {, } \\
& L_{1}=T_{a b c} E^{a b} X^{c}=\left(T_{111}+T_{112}\right) X^{1}+\left(T_{112}+T_{222}\right) X^{2} \\
& +\left(T_{113}+T_{223}\right) X^{3}, \\
& (1 / 2) C_{21}=T_{a_{1} a_{2} a_{3}} T_{b_{1} b_{2} b_{3}} A^{a_{1} b_{1}} A^{a_{2} b_{2}} X^{a_{3}} X^{b_{3}} \\
& =\left[T_{111} T_{122}-\left(T_{112}\right)^{2}\right]\left(X^{1}\right)^{2}+\left[T_{111} T_{222}-T_{112} T_{122}\right] X^{1} X^{2} \\
& +\left[T_{112} T_{222}-\left(T_{222}\right)^{2}\right]\left(X^{2}\right)^{2} \\
& +\left[T_{122} T_{113}+T_{111} T_{223}-2 T_{112} T_{123}\right] X^{1} X^{3} \\
& +\left[T_{112} T_{223}+T_{113} T_{222}-2 T_{122} T_{123}\right] X^{2} X^{3} \\
& +\left[T_{113} T_{223}-\left(T_{123}\right)^{2}\right]\left(X^{3}\right)^{2} \text {. }
\end{aligned}
$$

\section{REFERENCES}

1. C. M. Cramlet, The derivation of algebraic invariants by tensor algebra, this Bulletin, vol. 34 (1928), pp. 334-342.

2. T. L. Wade, $A$ note on subgeometries of projective geometry as the theories of tensors, this Bulletin, vol. 47 (1941), pp. 475-478.

3. H. Weyl, The Classical Groups. Princeton, 1939, p. 244.

4. R. Weitzenb"ck, Ueber affine Invarianten bei Kegelschnitten, Proceedings, Akademie van Wetenschappen, Amsterdam, vol. 43 (1940), pp. 159-167.

5. - Invariantentheorie, Groningen, 1923.

6. J. Thomae, Über orthogonale Invarianten der Curven dritter Ordung, Leipziger Berichte, vol. 51 (1899), pp. 317-353.

7. M. Stuyvaert, Point remarquable dans le plan d'une cubique, Nouvelles Annales de Mathématique, (3), vol. 20 (1899), p. 275.

8. C. E. Brooks, Orthic curves, Proceedings of the American Philosophical Society, vol. 43 (1904), p. 294.

9. H. S. White, Plane Curves of the Third Order, Cambridge, Mass., 1925, pp. 4751.

University of Alabama 